\section{Wrong intraocular lens events - what lessons have we learned? A review of incidents reported to the National Reporting and Learning System: 2010-2014 versus 2003-2010}

LR Steeples ${ }^{1}$, M Hingorani $^{2}$, D Flanagan ${ }^{2}$ and SP Kelly ${ }^{3}$

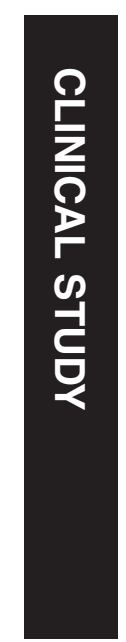

\begin{abstract}
Purpose To identify the causal factors in wrong intraocular lens (IOL) events from a national data set and to compare with similar historical data (2003-2010) prior to mandatory checklist use, for the purpose of developing strategies to prevent never events. Methods Data from wrong IOL patient safety incidents (PSIs) submitted to the National Reporting and Learning System (2010-2014) were reviewed by thematic analysis and compared with the data

of surgical checklists, wrong IOL incidents continue to occur and are probably underreported. Human or behavioural factors are heavily implicated in these errors and need to be addressed by novel approaches, including simulation training. There is also scope to further improve the quality and detail of incident reporting and analysis to enhance patient safety. Eye (2016) 30, 1049-1055; doi:10.1038/eye.2016.87; published online 13 May 2016
\end{abstract} previously collected by the group using the same methodology.

Results One hundred and seventy eight wrong IOL PSIs were identified. The contributory factors included: transcription errors $(n=26)$; wrong patient biometry $(n=21)$; wrong IOL selection $(n=16)$; changes in planned procedure $(n=16)$; incorrect IOL brought into theatre $(n=11)$; left/right eye selection errors $(n=9)$; communication errors $(n=9)$; and positive/negative IOL power errors $(n=9)$. In 44 PSIs, no causal factor was reported, limiting the learning value of such reports. Compared with the data from previous years, biometry errors were much reduced but IOL transcription and documentation errors were greater, particularly if further checks did not refer to the original source documentation. IOL exchange surgery was reported in 45 cases.

Conclusions The selection and implantation of the correct IOL is a complex process which is not adequately addressed by existing checking procedures. Despite the introduction
Cataract surgery with intraocular lens (IOL) implantation is one of the most frequent operations in the UK ${ }^{1}$ and a highly cost-effective treatment which improves quality of life. ${ }^{2}$ However, cataract surgery carries risk for errors that fulfil the definition of a so-called 'never event'. ${ }^{3}$ Never events are 'serious, largely preventable patient safety incidents that should not occur if the available preventative measures have been implemented'. ${ }^{3}$ Potential never events in cataract surgery include wrong patient, wrong eye, and wrong implant. Cataract surgery carries a greater risk of inserting a wrong implant than any other procedure requiring an implant. For every IOL procedure, multiple elements of the data must be measured accurately by specifically trained professionals using delicate instruments. IOL planning and selection requires analysis of many factors about the patient, the eye, their refractive desires, and previous ocular history as well as the use of complex formulae. Correct IOL

\section{Introduction}

\author{
'Manchester Royal Eye \\ Hospital, Central \\ Manchester Foundation \\ Trust, Oxford Road, \\ Manchester, UK
}

${ }^{2}$ Moorfields Eye Hospital NHS Foundation Trust, London, UK

\section{${ }^{3}$ Ophthalmology} Department, Royal Bolton Hospital, Bolton NHS Foundation Trust, Bolton, UK

Correspondence:

LR Steeples, Manchester Royal Eye Hospital, Central Manchester Foundation Trust, Oxford Road, Manchester M13 9WL, UK Tel: +44 (0)120439 0694; Fax: +44 (0)1204390694 E-mail: laura.steeples@cmft. nhs.uk

Received: 30 June 2015 Accepted in revised form: 6 April 2016

Published online:

13 May 2016 
selection requires the clinician to check multiple, often small font, biometry and other data fields on different sheets of paper or screens as well as ensuring all pertain to the correct eye and often to transcribe such IOL selection. This, together with a huge number of permutations of available IOL types and dioptric powers, within a high volume list, increases the risks far beyond that which one could expect with a hip replacement or similar implant. ${ }^{4}$ The evolving use of multi-focal and toric IOLs adds to the risk of error.

In recent years, the use of surgical checklists has been associated with reductions in surgical mortality and morbidity in developed and emerging economies ${ }^{5-7}$ and became mandatory in 2009 in NHS care in England and Wales. Cataract specific checklists were introduced in the US by the American Academy of Ophthalmology in $2009^{8}$ and in the UK by the Royal College of Ophthalmologists ('the College') in 2010.9

It is recognised that learning from patient safety incidents, through incident reporting, causal analysis and dissemination for wider learning, is a core process for improving care quality. ${ }^{10,11}$ In the UK, the National Patient Safety Agency (NPSA) and, subsequently, NHS England, have facilitated the collection of a national incident data set since 2003 through the National Reporting and Learning System (NRLS). ${ }^{12}$ In early 2010, a review of wrong IOL implantation incidents reported to the NRLS since 2003 was undertaken by one of the authors. ${ }^{13}$ The present analysis re-assesses wrong IOL events reported to the NRLS to determine whether the implementation of recent measures such as cataract checklists and never event reporting have led to improvement and to help develop further preventative strategies.

\section{Materials and methods}

A retrospective review of 'wrong IOL' patient safety incidents (PSIs) reported to the NRLS in the period 1 February 2010 to 31 May 2014 was undertaken. A search of the incident level patient safety data was performed using the following free text terms: 'wrong' or 'incorrect' or 'error' and associated with 'cataract' or 'lens' or 'intraocular' or 'IOL' or 'dioptre' or 'cataract surgery' or 'phaco' or 'phako' or 'biometry' or 'IOL master.'

Wrong IOL incident reports were extracted and analysed. We acknowledge that invariably causation in safety incidents is multifactorial. However, the data and free text in each incident was reviewed and scrutinised by thematic analysis to identify the major reason for error. The timing of error detection, management including any surgical intervention and the level of harm reported was also reviewed.

These data were compared with similar wrong IOL incident historical data, obtained from the NRLS by our group in the period 2003-2010, using the same search terms and analysis method. The previous data extraction reviewed 164 PSIs.

\section{Results}

In total, 178 wrong IOL PSIs were identified, reviewed and compared with the historical data. These are categorised in Table 1 by when the incident occurred within the cataract care pathway. In Table 2, details of system failures and unsafe human behaviours detailed in incident reports are presented.

\section{Causal factors}

The nature of the errors showed many similarities to the historical data. First, we identified recurrent problems in matching clinical documents with the correct patient or the correct eye. In particular, and of concern, misfiling of the biometric data in the wrong patient notes was reported in far more incidents $(21 / 178)$ than previously $(4 / 164)$ and there were persistent reports of using notes not matched to the patient (5).

Second, errors in the selection and documentation of the IOL to be implanted continue with recurring themes

Table 1 Major reason for wrong IOL implantation by when the incident occurred within the cataract care pathway: comparison of data from 2003-2010 to 2010-2014

\begin{tabular}{|c|c|c|}
\hline \multirow[t]{2}{*}{ Major reason for error } & \multicolumn{2}{|c|}{ Number of reports } \\
\hline & 2003-2010 & 2010-2014 \\
\hline \multicolumn{3}{|l|}{ Preoperative issues } \\
\hline Biometry error & 29 & 5 \\
\hline Transcription error & 14 & 26 \\
\hline \multicolumn{3}{|l|}{ Incorrect data for patient } \\
\hline Wrong patient biometry data & 4 & 21 \\
\hline Wrong patient medical notes & 2 & 5 \\
\hline Patient identification issues & 4 & 0 \\
\hline \multicolumn{3}{|l|}{ IOL selection errors } \\
\hline Wrong IOL selected & 21 & 16 \\
\hline Right/left eye confusion & 5 & 9 \\
\hline Plus/minus power error & - & 9 \\
\hline \multicolumn{3}{|l|}{ Intraoperative } \\
\hline \multicolumn{3}{|l|}{ Changes in planned procedure } \\
\hline Change in list order & 8 & 8 \\
\hline Selected IOL not available & 3 & 0 \\
\hline Complicated surgery & 3 & 8 \\
\hline \multicolumn{3}{|l|}{ Perioperative/others } \\
\hline Communication errors & 2 & 9 \\
\hline Handwriting misinterpretations & 7 & 4 \\
\hline Wrong IOL brought into theatre & & 12 \\
\hline Wrong IOL in wrong box & - & 1 \\
\hline No reason documented (\%) & $62(38)$ & $44(25)$ \\
\hline Total number of PSIs & 164 & 178 \\
\hline
\end{tabular}


from the previous review. IOL selection mistakes included using biometry data for the wrong laterality (wrong eye) and the surgeon making the incorrect selection for the planned refractive target. In one case, an unexpected change in the biometric data layout resulted in wrong IOL selection and, in a second, a poor quality printout with unclear data was misread by the surgeon.

In the earlier 2003-2010 review, transcription of the IOL choice from biometry data to another format was a leading source of error. In these data, mistakes in transcription were even more common (21), alongside unclear documentation of positive and negative dioptric powers such as ' +3 ' instead of ' -3 ' and mistakes such as '29' power misread as ' 19 '. The errors involved transcription onto white boards and, less commonly, paper sheets such as operating lists, and subsequent reference to this instead of using the original clinical or biometry documents. A particular risk within this category was transcription into an ordered list of dioptric powers written on white boards when there was a change in list order.

The number of reports with inaccurate biometry is far smaller than the number observed in the previous review (5 versus 29). Similar to that reported previously, failure to remove rigid contact lens in adequate time before biometry measurements were taken is a rare cause (1).

\section{Change in planned procedure or change in team members during list}

A change in planned procedure, owing to list order change or intraoperative complication, particularly when a different IOL model or power had to be selected, was implicated in a number of incidents (16). Changes in staff members during the list, often with consequent breakdown in communication, were detailed in some reports as contributing factors.

\section{Electronic patient records}

A number of reports (17) detailed errors with respect to the use of electronic patient records (EPRs), which were not identified in the earlier analysis. These incidents related to accessing incorrect patient records or incorrect eye data, resulting in failure to match the correct patient or eye with the correct data. These mistakes were carried downstream in the surgical pathway by transcribing the incorrect selection with subsequent checks failing to refer to source EPR documents. Incorrect biometry data in the EPR following manual entry errors or incorrect electronic import were also described.

\section{Timing of discovery of error}

The majority of errors were detected after completion of surgery (Figure 1a) with 17 promptly identified when completing the operating note. Unexpected refractive outcomes (so-called 'refractive surprise') and reduced visual acuity were the leading causes for identification of error postoperatively.

\section{Level of harm}

Reporting the level of harm is mandatory (Figure 1b). In the 45 cases that underwent further surgical intervention no harm was the commonest classification (26) with

Table 2 Examples of potentially unsafe systems and human behaviours described in reports

- Checklist procedure failing to recognise non-matched patient and data including incorrect notes or incorrect biometry with patient

- Transcribing IOL selection onto white boards, theatre lists and paper notes and not checking intraoperatively with source documents

- Writing lens selection on whiteboard for the next case during an on-going operation

- Failure to refer to source documents

- Surgeon selecting IOL from memory and ignoring notes

- Unclear handwriting or notation of plus/minus status of lens power

- Stockpiling lenses for all cases on the list in theatre

- Not challenging surgeon despite concerns about IOL selected

- Undermining or ignoring established safety procedures and protocols
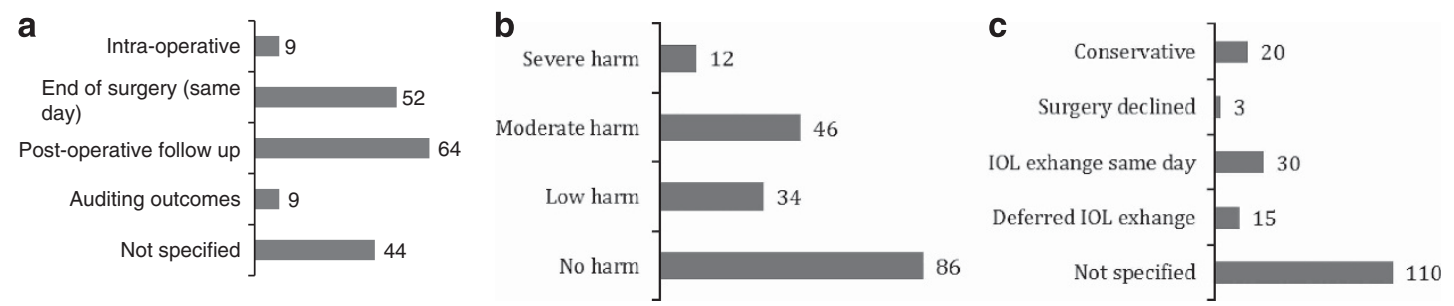

Figure 1 (a) Reported timing of the detection of wrong IOL implantation error. (b) Reported classification of the level of harm caused by the wrong IOL incident. (c) Reported management of the wrong IOL incident. 
reports of low, moderate, and severe harm in 4, 12, and 3 reports, respectively. The basis for these categorisations was not clear from the information reported.

\section{Management of errors}

In 68 reports, the further management of the wrong IOL event was documented. (Figure 1c) Conservative management was most often chosen if the visual outcome (unaided or with spectacle correction) was acceptable to the patient. IOL exchange (45/68 reports) was the only reported further surgical intervention with no reports of other management such as laser refractive correction or secondary 'piggyback' IOL implantation. A clinical adverse outcome and indication for further surgery was documented in only 4 cases with refractive surprise (3) and predicted unacceptable outcome (1) detailed. Unfortunately, the reason for further surgery (such as significant refractive surprise or specific nature of any patient harm) was not clearly outlined in the remaining majority (41) of reports. Adverse outcomes from further surgical intervention were described in four cases with retinal tear (1), corneal decompensation (2), and vision loss (1; duration unknown) reported.

\section{Data comparisons}

The number of wrong IOL incidents recorded in this investigation (178 incidents during 2010-2014) exceeds the number reported in our previous analysis covering reporting over a longer time period (164 incidents during 2003-2010). The annual volume of cataract surgery procedures has increased somewhat over the time period 2003-2014: the number of operations performed in the NHS in England in 2003, 2010, and 2013 was 298 504, 328 504, and 358143 , respectively. ${ }^{14}$

However, attempts to directly compare the rate of wrong IOL events between these two periods can be misleading. The true incidence of wrong IOL implantation is unknown as incident reporting systems do not necessarily provide an accurate assessment of the epidemiology of medical errors ${ }^{15,16}$ and mandatory reporting of incidents since 2010 alongside a better reporting culture may explain the apparent higher rate in the later data.

\section{Discussion}

Wrong IOL implantation has been included as a never event since 2011 if the incident requires surgical intervention and/or if the patient suffers complications. ${ }^{17}$ From 2015 all wrong IOLs fulfil the revised definition of never event. ${ }^{18}$ This change is likely to result in ophthalmic surgery being one of the most frequent sources of surgical never events in the UK.

There is a debate as to whether wrong IOL incidents truly fulfil the spirit of a never event ${ }^{19}$ given that most patients do well. Furthermore, reputational damage and financial penalties associated with never events may lead to under-reporting. ${ }^{8,20}$ However, that wrong IOLs or refractive surprise are a problem cannot be argued. Surgical never events are the most frequently reported type of never event ${ }^{21}$ and wrong IOL implants are the leading cause of wrong implant never events. ${ }^{22}$ Errors in cataract care are a common cause of litigation in ophthalmic care ${ }^{23}$ with wrong IOL insertion the single commonest $(19 \%)$ cause. ${ }^{24}$ In addition, there is little doubt that the never event system has raised awareness and led to changes in practice to improve safety. ${ }^{20,25,26}$

\section{Causal factors and addressing them}

The present data demonstrate the persistence of wrong IOL events in the NHS in England and Wales, despite promotion of patient safety and mandatory use of surgical checklists. A recent analysis of wrong IOL incidents (44 cases) from the Veterans Health Administration confirms a similar situation in the USA. ${ }^{20}$ Analysis of the causal factors within our data and elsewhere suggests that non-technical errors (human factors) are the predominant cause of mistakes. ${ }^{27}$

Surgical checklists have been widely adopted, and are mandatory in many healthcare systems, following demonstration of improvement in surgical morbidity and mortality. ${ }^{5-7}$ It may be that, despite a bespoke cataract checklist being in widespread use, IOL selection could be further honed for greater safety locally with greater emphasis on team function and practical use. ${ }^{28}$ Local tailoring of surgical safety checklists and protocols, with active engagement from clinicians and staff, is highly encouraged, with evidence suggesting greater success and sustained use through this approach. ${ }^{29,30}$ Experience demonstrates that checklists alone are not completely effective in preventing adverse incidents ${ }^{31,32}$ and recent publications question the assumption that adoption of checklists routinely result in better surgical safety outcomes. ${ }^{33,34}$ It is increasingly recognised that multiple factors, such as staff engagement and organisational support, influence checklist use and adherence, and this may help explain variation in reported outcomes with checklists. ${ }^{34,20}$ The present analysis also adds to the view that checklist adoption is not enough.

Human errors and team behaviour provide actionable targets for improving safety and can be addressed positively by training using realistic immersive simulation in both medical and non medical fields. ${ }^{16,35-37}$ The surgical safety process needs to be completed by teams who have 
trained together and had appropriate education in human factors in safe team work including communication, situation awareness, decision making, leadership and stress management. ${ }^{31,38}$

We have provided a series of recommendations in Table 3, with specific guidance for strategies during the recognised vulnerable stages of the surgical care pathway ${ }^{31}$ and to address unsafe behaviours evident in our incident data (Table 2). The recent recommendations from National Standards for Invasive Procedures ${ }^{31}$ (NatSSIPs) also focus on robust and consistent approaches by the whole surgical team throughout the procedure. In particular, we suggest team simulation training with scenarios involving common mistakes, such as wrong patient biometry, alongside specific exercises for staff to gain confidence in areas such as dealing with a change or challenging concerns. We believe simulation training is an important, novel approach to improve these skills in cataract surgery teams.

\section{Reporting quality}

The determination of true levels of harm and the ability to learn are undermined by a continuing lack of detail and consistency found in the incident reports in this study. For example, the NPSA definitions of harm indicate a return to surgery without permanent harm should be coded as 'moderate' severity, yet the majority of cases undergoing IOL exchange were graded as 'no harm.' 39 Reports contained no details of causal factors in $25 \%$ of the 2010-2014 data and in 38\% of the 2003-2010 data. We recommend that reporting improves in this respect and reporters are encouraged to refer to the definitions of harm as defined in NPSA's Seven Steps to Patient Safety. ${ }^{31,39}$

\section{Conclusion}

This analysis of national PSI data from England and Wales demonstrate that wrong IOL episodes continue to occur in NHS care despite the widespread use of bespoke checklists in cataract surgery and we recommend that there should be a focus on human factors and team-based simulation training, although who will develop, fund, and resource such training is uncertain. We encourage local reporters of patient safety incident reports to be more detailed and to be more accurate about levels of harm and to include root-cause analysis reporting to ensure better

Table 3 Recommendations of important principles to adhere to in standard operating protocols for correct IOL implantation

\section{Recommendations}

Consistent checks and no assumptions: three-stage approach to the sign in, time out, and sign out checks:9,31 (i) identity and document check; (ii) eye (left or right) check and (iii) IOL check (power, type, and model) repeated at each stage. Specifically check all documents, especially biometry data matches the patient and operated eye at each stage.

IOL selection: always refer to source biometry and clinical documents during IOL checks at each stage listed above. Any unusual powers or models or negative powers voiced during the 'team brief' and 'time out' stages. Always check the selection is made using the correct formula, A-constant and pertains to correct eye.

Transcriptions: to avoid mistakes and cascading of errors:

(i) no writing of multiple IOL selections onto white boards or theatre lists and the transcription should always be matched to a single patient and their identifying data; (ii) any minus lens powers clearly denoted by the word 'minus' and (iii) transcription onto only locally agreed IOL selection sheets (paper or electronic) and clear handwriting is crucial.

Avoid re-selection during procedure: availability of the selected IOL always confirmed before patient enters theatre and the start of procedure.

Lens collection: (i) IOL only selected from the lens bank once staff and surgeon have confirmed the selection, (ii) only one IOL in theatre with the patient and where the lens bank is in the theatre for a single lens to be selected and removed as suggested and be positioned in a selected place as per local protocol away from the lens bank (no stockpiling).

Change: if change in list order or procedure, entire team pause and repeat brief. If change in staffing during list/procedure: pause, repeat brief, and if new staff involved in IOL selection or collection repeat checks.

Re-selection: if need to reselect different IOL during the procedure: entire team pauses, remove the original IOL from theatre, and repeat process for selection of lens including identity, eye, and IOL check.

Challenge and check: staff encouraged and allowed to challenge any issues, concerns or inconsistencies regarding IOL selection immediately.

Simulation training: scenario-based team training exercises to learn local checklist protocol, improve repeatability, develop nontechnical skills and aid recognition of common mistakes (see Table 2 for possible scenarios). 
national learning. We provide recommendations of important principles to adhere to in standard operating protocols for correct IOL implantation and we encourage the local development and adoption of such protocols. Examples of protocols in use at the author's institutions and other patient safety information is available on the College's website. ${ }^{40}$

\section{Summary}

What was known before

- Wrong IOL implantation is a serious patient safety incident and is defined by NHS England as a 'never event'.

- Learning from patient safety incidents and failures, through incident reporting, causal analysis, and dissemination for wider learning, is a core process for improving patient care quality.

\section{What this study adds}

- Despite the introduction of surgical checklists and major patient safety initiatives, wrong IOL incidents continue to occur and are probably under-reported.

- Human or behavioural factors remain heavily implicated in wrong IOL incidents and need to be addressed through further training and we suggest the importance of simulation training.

- $\quad$ Recommendations of important principles to adhere to are provided.

\section{Conflict of interest}

The authors declare no conflict of interest.

\section{Acknowledgements}

We are grateful to the staff at the National Reporting and Learning System for data extraction.

\section{References}

1 Health and Social Care Information Centre. Hospital episode statistics admitted patient care England 2013-2014. Available at http://www.hscic.gov.uk/catalogue/PUB1 6719/hosp-epis-stat-admi-summ-rep-2013-14-rep.pdf (accessed 26 November 2015).

2 Agarwal A, Kumar DA. Cost effectiveness of cataract surgery. Curr Opin Ophthalmol 2011; 22: 15-18.

3 National Patient Safety Agency. Never Events Framework 2009/2010. National Patient Safety Agency: London, 2009. Available at www.nrls.npsa.nhs.uk/neverevents/? entryid45 $=59859$ (accessed 26 November 2015).

4 Neily J, Mills PD, Eldridge N, Carney BT, Pfeffer D, Turner JR et al. Incorrect Surgical procedures within and outside of the operating room: a follow-up report. Arch Surg 2011; 146: $1235-1239$.

5 Haynes AB, Weiser TG, Berry WR, Lipsitz SR, Breizat AH, Dellinger EP et al. A surgical safety checklist to reduce morbidity and mortality in a global population. $N$ Engl J Med 2009; 360: 491-499.
6 de Vries EN, Prins HA, Crolla RM, den Outer AJ, van Andel G, van Helden $\mathrm{SH}$ et al. Effect of a comprehensive surgical safety system on patient outcomes. N Engl J Med 2010; 363: 1928-1937.

7 Neily J, Mills PD, Young-Xu Y, Carney BT, West P, Berger DH et al. Association between implementation of a medical team training program and surgical mortality. JAMA 2010; 304: 1693-1700.

8 Lum F, Schachat AP. The quest to eliminate never events. Ophthalmology 2009; 116: 1021-1022.

9 National Patient Safety Agency. WHO Surgical Safety Checklist: For Cataract Surgery Only. National Patient Safety Agency: London, 2010 Available at http://www.nrls.npsa.nhs.uk/ resources/clinical-specialty/surgery/?entryid $45=74132$.

10 Department of Health. An Organisation With a Memory. Department of Health: London, 2000 Available at http:// webarchive.nationalarchives.gov.uk/20130107105354/ http:/ / www.dh.gov.uk/prod_consum_dh/groups/dh_ digitalassets/@dh/@en/documents/digitalasset/dh_ 4065086.pdf.

11 Kelly SP. Guidance on patient safety in ophthalmology from the Royal College of Ophthalmologists. Eye 2009; 23: 2143-2151.

12 NHS England. National Reporting and Learning System. NHS England: London, Available at https://report.nrls.nhs.uk/ nrlsreporting/.

13 Kelly SP, Jalil A. Wrong intraocular lens implant; learning from reported patient safety incidents. Eye 2011; 25: 730-734.

14 Health and Social Care Information Centre. Hospital episode statistics admitted patient care England 2010, 2013 and 2014. Available at http:/ / www.hscic.gov.uk/article/2021/WebsiteSearch?q=title $\% 3 a+\% 22$ hospital+outpatient+activity $\% 22 \&$ sort $=$ Most + recent\&size $=10 \&$ page $=1 \&$ area $=$ both\#top.

15 Sari AB, Sheldon TA, Cracknell A, Turnbull A. Sensitivity of routine system for reporting patient safety incidents in an NHS hospital: retrospective patient case note review. BMJ 2007; 334: 79.

16 Panesar SS, Noble DJ, Mirza SB, Patel B, Mann B, Emerton M et al. Can the surgical checklist reduce the risk of wrong site surgery in orthopaedics?-Can the checklist help? Supporting evidence from analysis of a national patient incident reporting system. J Orthop Surg Res 2011; 6: 18.

17 NHS England. Never Events List 2013/14 update. NHS England: London, 2013 Available at http://www.england.nhs.uk/wpcontent/uploads/2013/12/nev-ev-list-1314-clar.pdf.

18 NHS England. Never Events list 2015/2016. NHS England: London, 2015. http:/ /www.england.nhs.uk/wp-content/ uploads/2015/03/never-evnts-list-15-16.pdf.

19 Hingorani M, Flanagan D. Wrong IOLs and never events in ophthalmology. College News 2014.

20 Neily J, Chomsky A, Orcutt J, Paull DE, Mills PD, Gilbert C et al. Examining wrong eye implant adverse events in the Veterans Health Administration with a focus on prevention: a preliminary report. J Patient Saf 2015; e-pub ahead of print 16 March 2015.

21 NHS England. Standardise, educate, harmonise. Commissioning the conditions for safer surgery Summary of the report of the NHS England Never Events Taskforce. NHS England: London, 2014. Available at http://www.england.nhs.uk/wpcontent/uploads/2014/02/sur-nev-ev-tf-sum-rep.pdf.

22 NHS England. Never Events Reported As Occurring Between 1 April 2013 and 31 March 2014. NHS England: London, 2014. Available at http://www.england.nhs.uk/wpcontent/uploads/2014/12/2013-14-NE-data-FINAL.pdf. 
23 Ali N. A decade of clinical negligence in ophthalmology. BMC Ophthalmol 2007; 7: 20.

24 Mathew RG, Ferguson V, Hingorani M. Clinical negligence in ophthalmology: fifteen years of national health service litigation authority data. Ophthalmology 2013; 120: 859-864.

25 Zamir E, Beresova-Creese K, Miln L. Intraocular lens confusions: a preventable "Never Event"- The Royal Victorian Eye and Ear Hospital Protocol. Surv Ophthalmol 2012; 57: 430-447.

26 The Royal College of Ophthalmologists Significant Events in Cataract Surgery. The Royal College of Ophthalmologists: London. Available at https:/ / www.rcophth.ac.uk/professio nal-resources/revalidation/clinical-sub-specialties/cataract /significant-events-in-cataract-surgery/.

27 Azuara-Blanco A, Reddy A, Wilkinson G, Flin R. Safe eye surgery: non-technical aspects. Eye 2011; 25: 1109-1111.

28 Sendlhofer G, Mosbacher N, Karina L, Kober B, Jantscher L, Berghold A et al. Implementation of a surgical safety checklist: interventions to optimize the process and hints to increase compliance. PLOS ONE 2015; 10: e0116926.

29 Gillespie BM, Marshall A. Implementation of safety checklists in surgery: a realist synthesis of evidence. Implement Sci 2015; 10: 137.

30 NHS England Patient Safety Domain. Patient Safety Alert. Stage 2, supporting the introduction of the National Safety Standards for Invasive Procedures 2015. Available at http:/ / www. england.nhs.uk/wp-content/uploads/2015/09/psa-natssips. pdf (accessed 26 November 2015).

31 NHS England Patient Safety Domain. National Safety Standards for Invasive Procedures 2015 Available at http:/ / www.england.nhs.uk/patientsafety/wp-content/uploads / sites/32/2015/09/natssips-safety-standards.pdf (accessed 26 November 2015).

32 Paull DE, Mazzia LM, Neily J, Mills PD, Turner JR, Gunnar W et al. Errors upstream and downstream to the Universal
Protocol associated with wrong surgery events in the Veterans Health Administration. Am J Surg 2015; 210: 6-13.

33 Urbach DR, Govindarajan A, Saskin R, Wilton AS, Baxter NN. Introduction of surgical safety checklists in Ontario, Canada. N Engl J Med 2014; 370: 1029-1038.

34 Gagliardi AR, Straus SE, Shojania KG, Urbach DR. Multiple interacting factors influence adherence, and outcomes associated with surgical safety checklists: a qualitative study. PLoS One 2014; 9: e108585.

35 Helmreich RL, Merritt AC, Wilhelm JA. The evolution of crew resource management training in commercial aviation. Int J Aviat Psychol 1999; 9: 19-32.

36 Holzman RS, Cooper JB, Gaba DM, Philip JH, Small SD, Feinstem D. Anesthesia crisis resource management: real-life simulation training in operating room crises. J Clin Anesth 1995; 7: 675-687.

37 Cook DA, Hatala R, Brydges R, Zendejas B, Szostek JH, Wang AT et al. Technology-enhanced simulation for health professions education: a systematic review and metaanalysis. JAMA 2011; 306: 978-988.

38 Hempel S, Maggard-Gibbons M, Nguyen DK, Dawes AJ, Miake-Lye I, Beroes JM et al. Wrong-site surgery, retained surgical items, and surgical fires: a systematic review of surgical never events. JAMA Surg 2015; 150: 796-805.

39 National Patient Safety Agency. Seven Steps to Patient Safety. National Patient Safety Agency: London, 2004. Available at http:/ / www.nrls.npsa.nhs.uk/resources/collections/sevensteps-to-patient-safety/?entryid45 $=59787$.

40 Patient Safety Information. Checklist Examples. The Royal College of Ophthalmologists 2015. Available at https:// www.rcophth.ac.uk/standards-publications-research/pati ent-safety-information/ (accessed 26 November 2015). 